\title{
Evaluación de la primera jornada del Foro de Investigación Educativa de la REDIECH
}

\author{
María Silvia Aguirre LaresEva \\ América Mayagoitia Padilla \\ Profesoras investigadoras \\ Centro de Investigación y Docencia \\ Universidad Pedagógica Nacional, Unidad 081
}

\section{Resumen}

S e reseña la primera jornada del Foro de Investigación Educativa de la Rediech, cuyos propósitos están encaminados a fortalecer la formación para la investigación educativa. La evaluación del desarrollo de las actividades se realiza con el fin de orientar las decisiones para la mejora de las siguientes jornadas de trabajo. La valoración se realiza a partir del análisis de la información contenida en diversos instrumentos como el registro de asistencia, una encuesta, relatorías y opiniones de participantes. Los resultados se organizan en tres apartados: organización del evento, descripción de la población asistente y opiniones sobre la calidad académica de las actividades. En términos generales las opiniones fluctuaron entre muy bien y excelente, en lo que se refiere al desarrollo de la jornada, aunque algunos detalles merecen una mayor aplicación organizativa como el manejo de los tiempos y el clima participativo.
Palabras clave: formación para la investigación, evaluación, redes

La Red de Investigadores Educativos Chihuahua (REDIECH) reconoce la necesidad de evaluar permanentemente su proyecto académico, así como la organización institucional que le da sustento. Una de las tareas centrales que la Red se encuentra desarrollando en la actualidad, es un proyecto aprobado y apoyado por el Fondo Mixto de Fomento a la Investigación Científica y Tecnológica Conacyt-Gobierno del estado de Chihuahua. Dicho proyecto tiene como propósito fortalecer la REDIECH a través de diferentes actividades. Una de ellas se denomina Foro de Investigación Educativa el cual tiene como objetivos:

- Construir un espacio que permita el intercambio de saberes y prácticas entre las personas interesadas en la investigación educativa.

- Coadyuvar a la formación de profesionistas educativos de alta calidad. 
- Promover la reflexión en torno a las reflexiones epistemológicas, teóricas y metodológicas de la práctica de la investigación educativa, en particular la que se desarrolla en el estado de Chihuahua.

- Facilitar herramientas para la asesoría y desarrollo de proyectos de investigación, así como para la formación de grupos de investigadores en los posgrados.

El Foro se desarrollará en seis jornadas de trabajo, distribuidas en los dos años de vigencia del proyecto ante Fondos Mixtos ConacytGobierno del Estado de Chihuahua. El día 14 de agosto de 2010, se realizó la primera jornada del Foro, misma que trató el tema "Debates epistemológicos en torno a la investigación educativa”. La jornada se extendió por nueve horas presenciales, además de las que cada participante destinó al estudio de los materiales de lectura sugeridos para abordar la temática.

En el proceso evaluativo, se adopta, una perspectiva de "Evaluación para la mejora académica" cuya nota distintiva es la emisión de juicios valorativos fundamentados que orienten la toma de decisiones que conducen a la realización de acciones de mejora de los programas, de las condiciones, de los grupos y los individuos (Gobantes, 2001).

Esta perspectiva de evaluación, permite el crecimiento cualitativo de las instituciones al propiciar la generación de círculos virtuosos donde sus integrantes promueven la evaluación del trabajo desempeñado en un proyecto o actividad, como base fundamental para retroalimentar positivamente su actuar institucional en busca del logro de los objetivos planteados.

Los valores que orientan los procesos de evaluación para la mejora son: el respeto irres- tricto a los usuarios en tanto son ellos mismos quienes valoran la calidad de los servicios que reciben, la transparencia en la rendición de cuentas en la que las instituciones están abiertas a que se opine sobre cómo se hacen las cosas, de tal forma que sea posible el diseño permanente de agendas de mejoramiento y el compromiso que busca beneficios sociales, que en este caso están orientados a mejorar la calidad educativa a través del impulso a uno de sus ejes fundamentales: la generación y difusión del conocimiento científico en educación.

En congruencia con esta posición, en este ejercicio evaluativo se valoran los elementos fundamentales que orientaron la organización y desarrollo de la jornada "Debates Epistemológicos de la Investigación Educativa” buscando identificar sus fortalezas y debilidades, lo que sin duda contribuye a la mejora organizacional de la REDIECH en tareas futuras. La valoración se realiza gracias a la sistematización de la información contenida en la ficha de inscripción, los resultados obtenidos en la aplicación de una encuesta de opinión a los asistentes al evento, las opiniones vertidas a partir de una guía cualitativa que se hizo llegar a informantes clave, las relatorías de las mesas de discusión y las del grupo de discusión que se realizó con la comisión organizadora del evento.

\section{Acerca de la organización del evento}

El primer aspecto refiere que la convocatoria no solo fue emitida por la ReDIECH, a ella concurrieron instancias diversas, en calidad de instituciones participantes: el Centro de Investigación y Docencia, la Universidad Pedagógica Nacional, Unidad 081, el Departamento de Formación y Actualización de Docentes y el 
Departamento de Investigación Educativa de la Secretaría de Educación y Cultura, el Sindicato Nacional de Trabajadores de la Educación Sección 8, la escuela Normal Rural "Ricardo Flores Magón", la Universidad Autónoma de Chihuahua División Posgrado, la Escuela Normal Superior "José E. Medrano", el Instituto de Pedagogía Crítica AC y el Tecnológico de Monterrey Campus Chihuahua. De inicio esto expresa un fuerte interés tanto en el trabajo de la REDIECH, como en el Foro en lo particular.

Gracias al presupuesto autorizado por Fondos Mixtos Conacyt-Gobierno del Estado de Chihuahua y las gestiones propias de la REDIECH, durante la primera jornada del Foro se proporcionaron los insumos materiales y la infraestructura adecuada para el desarrollo de los trabajos. Varios de los y las asistentes manifiestan que la atención recibida fue adecuada, se valora en los materiales proporcionados, en la amplitud de los espacios, la disponibilidad de estacionamiento para vehículos, los refrigerios y la comida. Por otra parte, se hacen señalamientos sobre las condiciones del lugar donde se desarrollaron las mesas de trabajo: la falta de lugares aislados y con ello el excesivo ruido ambiental, que interfirieron significativamente en la actividad

La difusión del evento fue cubierta por diferentes medios: entrevistas en radio, televisión y periódicos, además de las notas informativas que se estuvieron emitiendo en las semanas anteriores a la fecha del evento. Circulación de correos electrónicos, invitación a través de la página web de la REDIECH y presencia con autoridades educativas, fueron formas de extender la invitación. Con todo este esfuerzo, aún hay señalamientos que la invitación se circunscribió al ámbito educativo y que entonces, conviene ampliarla a otras áreas como escuelas de psicología, antropología, posgrados particulares, entre otros.

La organización de la jornada fue todo un reto a nivel operativo. La Comisión Organizadora se enfrentó a las limitantes de tiempo, vale la pena mencionar que las cuatro personas que conforman el núcleo organizativo, tienen además carga completa en sus instituciones de adscripción. Esta limitación fue superada con disposición y experiencia, además fue fundamental la solidaridad de integrantes de la REDIECH que se sumaron en diferentes momentos de la organización. Con todo, la Comisión Organizadora juzga que la realización de las siguientes jornadas del Foro debe fortalecerse con la inclusión de nuevos colaboradores.

Entre las observaciones a la parte organizativa, hay quienes proponen una mayor aplicación en la conducción del evento, sobre todo la fluidez del programa y la presentación de invitados y ponentes. Ello nos lleva a eficientar los canales de comunicación para poder afrontar los imprevistos a los que todo evento está expuesto.

\section{Acerca de los y las participantes}

Gracias a la organización, la inscripción registrada rebasó las expectativas iniciales que partieron del supuesto de contar con una participación máxima de 60 personas. Se contó con la asistencia de un total de 82 educadores, cuya presencia permite evidenciar el creciente interés de los profesionales de la educación, por adquirir las herramientas necesarias para desarrollar tareas de investigación educativa, lo que a su vez denota la urgencia de promover políticas que impulsen esta actividad en la entidad. 
De los asistentes, el 59\% son mujeres y el $41 \%$ hombres. Estos datos sugieren una mayoritaria incursión de las mujeres en la profesión educativa, sea en calidad de profesionista trabajadora o como estudiante de los posgrados que se ofrecen en la entidad.

Sobresale así mismo, que los y las asistentes mayoritariamente viven en la ciudad de Chihuahua (80\%); mientras que un $20 \%$ provienen de

Asistentes Inscritos, por sexo

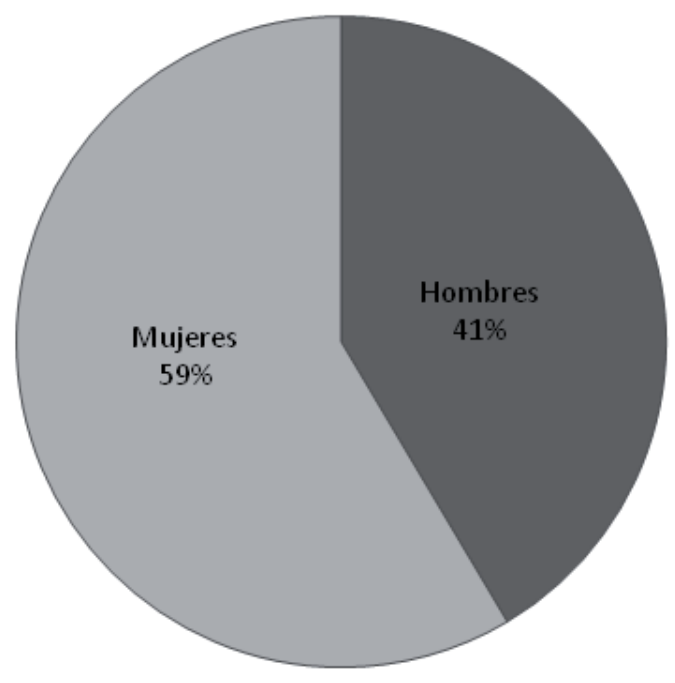

otras ciudades del estado. La presencia de participantes de ciudad Juárez, Parral, Delicias, Camargo, Nuevo Casas Grandes y Saucillo resulta muy alentadora si se consideran los esfuerzos que se debieron realizar para asistir al Foro, por la distancias de sus ciudades de residencia a la sede del evento (de dos a cuatro horas de traslado) y con las implicaciones familiares y económicas inherentes.

Finalmente, es necesario resaltar que del total de asistentes, menos de un $30 \%$ son miem-

\section{Asistentes inscritos, procedencia}

$\square$ Ciudad de Chihuahua $\quad \square$ Otra

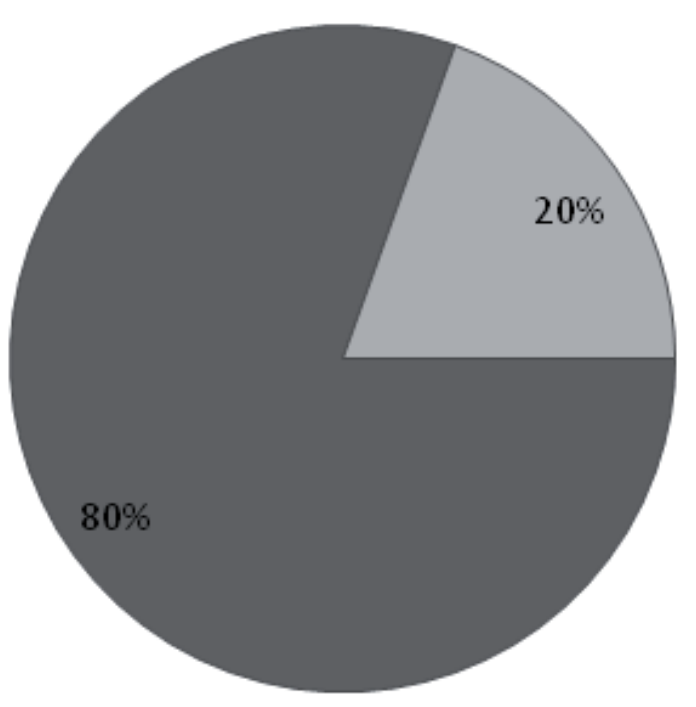

bros de la ReDiech, lo que permite reconocer la capacidad de convocatoria de la red; pero además, el potencial crecimiento de la misma y el interés de grupos de profesionales en ampliar y compartir sus experiencias sobre la investigación educativa.

La Rediech se enfrenta al reto de cumplir con su compromiso de constituirse en un organización que promueve la cultura de la investigación educativa en la entidad, a través de la generación y difusión del conocimiento científico en educación, de la formación académica en y para la investigación y la innovación, y en la generación de redes investigativas a nivel local, nacional e internacional. 


\section{Asistentes Inscritos, por membresía}

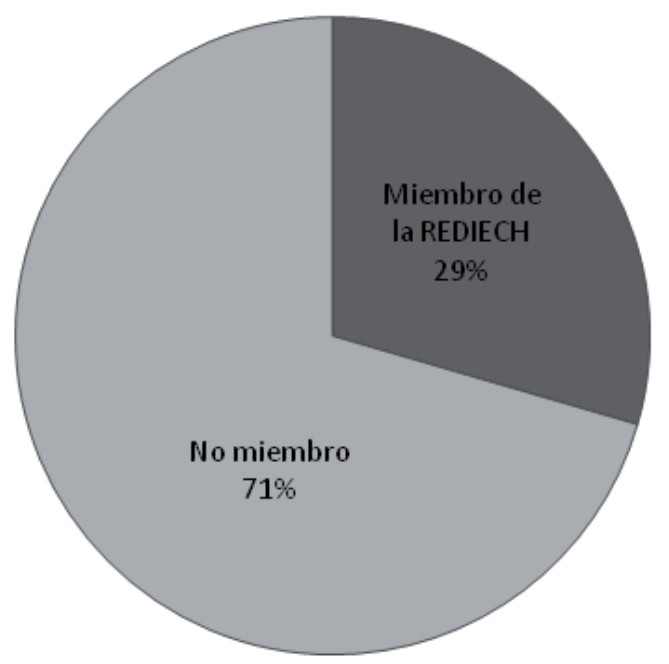

\section{La calidad académica del Foro}

El Foro incluyó tres actividades básicas: una conferencia magistral, un panel, y el trabajo en mesas de discusión. Para conocer el impacto de las actividades de la jornada se aplicó un cuestionario que consideraba cinco rangos de opinión: excelente, muy bueno, bueno, deficiente y pésimo. En los 10 ítems que respondieron las y los asistentes, la valoración fluctúa entre excelente y muy bueno; las opiniones sobre regular son escasas y solo una emite una apreciación deficiente. Por este comportamiento de las opiniones, de manera general se puede afirmar que la jornada cubrió las expectativas de la mayoría de la asistencia. Sin embargo, se perciben valoraciones diferenciadas según las actividades académicas y lo que ello implicó, como la organización, el uso del tiempo y los materiales. Estas apreciaciones se detallan a continuación.

a. El tema de la jornada fue valorado como excelente por tres de cada cuatro asistentes, el resto opina que fue muy bueno. En las opinio- nes se planteó que aunque el tema es complejo, su abordaje didáctico les permitió reflexionar sobre los fundamentos epistemológicos y ontológicos inherentes al trabajo de investigación educativa. Pareciera que el reto de reflexionar sobre la cientificidad de la investigación y las estrategias de generación de conocimiento, atraen fuertemente a quienes se encuentran transitando un proceso formativo y también a las y los académicos de educación superior.

b. La primera actividad del día fue la Conferencia Magistral "De la epistemología a la praxis educativa” que fue impartida por el Doctor Jorge Mario Flores Osorio. Esta fue la actividad más relevante para quienes asistieron al Foro; las expresiones parten de lo complejo de la temática y por ello ponderan el estilo didáctico, la sencillez humana del conferencista, la profundidad en el tratamiento del tema, el manejo del tiempo y la disponibilidad para intercambiar opiniones. Para el 69\% la exposición fue excelente, el resto opina que muy buena. En cuanto al contenido de la conferencia un $74 \%$ considera que fue excelente, solo una persona

\section{¿La exposición del conferencista le pareció?}

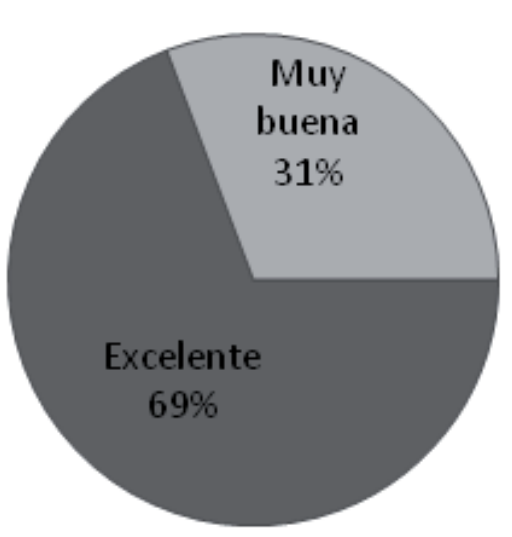


(el 3\%) opina que fue regular y el resto lo valora como muy bueno.

c. La segunda actividad del día consistió en un Panel que desarrolló el tema "El conocimiento científico, la polémica actual". Los tres panelistas son catedráticos de educación superior de instituciones de la ciudad de Chihuahua. La dinámica incluyó, además de la exposición de los ponentes, el debate de ideas entre los mismos, se consideraba que al término de este intercambio se dieran las participaciones de los y las asistentes. Sin embargo, estas participaciones fueron escasas ya que el debate entre panelistas sobrepasó el tiempo estimado.

Los juicios de valor emitidos en torno al panel dicen que solo el 34\% consideraron excelente el tema; en cuanto a la participación de los panelistas, los y las asistentes bajaron aún más su valoración ya que tan sólo el $28 \%$ la califica como excelente. Aunque más del $60 \%$ pondera como muy bueno tanto el tema como el desempeño de los panelistas, los resultados indican que fue una actividad que impactó menos en el ánimo de la asistencia, en comparación con la conferencia. Estas opiniones se refuerzan con las provenientes del cuestionario cualitativo y el grupo de discusión. Les pareció excesivo el intercambio entre panelistas y faltó establecer las relaciones o relevancia del tema con la investigación educativa, sobre todo lo más penalizado fue el manejo del tiempo que impidió una más amplia participación de la asistencia.

d. La última actividad del día consistió en el trabajo colectivo organizado en Mesas de Discusión que tuvieron el propósito de abrir el debate sobre el tema de la jornada. Este fue considerado el escenario participativo fundamental, la pretensión era analizar las ideas expuestas

\section{¿El trabajo de los panelistas le pareció?}

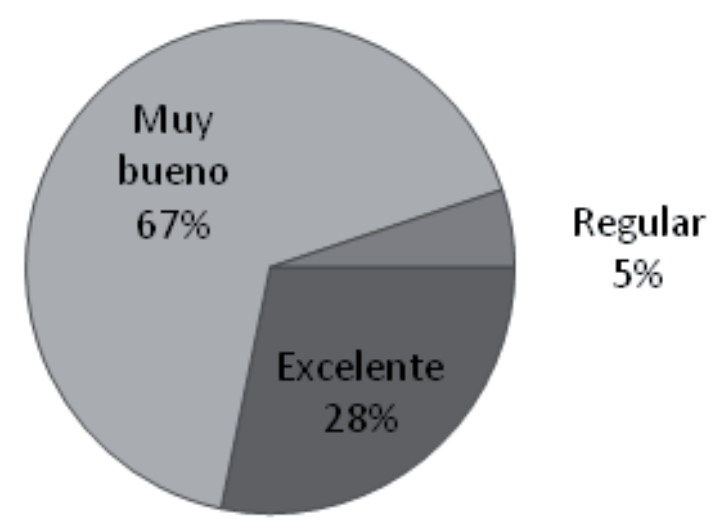

por los especialistas, además de compartir las ideas y las experiencias propias. Para ello, se organizaron tres mesas de discusión que trabajaron en torno a preguntas generadoras, aunque quienes las coordinaron tuvieron el cuidado de no constreñir la participación de los y las asistentes.

Las relatorías de las mesas muestran los tópicos más relevantes para la asistencia. Sobresalen las opiniones respecto a las dificultades para realizar investigación educativa (institucionales, de recursos, de tiempo); el poco impacto que tienen los hallazgos en la política educativa estatal; las limitaciones para establecer grupos de trabajo interinstitucionales; la escasa difusión de los resultados y la poca presencia de investigadoras/es de nuestra entidad en instancias u organismos nacionales e internacionales.

Otro bloque de participaciones se orientó hacia la reflexión sobre los contenidos de la conferencia y del panel, además del esfuerzo de encontrarle sentido en relación a los objetos de estudio que les interesa. Se compartieron expe- 


\section{¿El trabajo desarrollado en las mesas de discusión fue?}

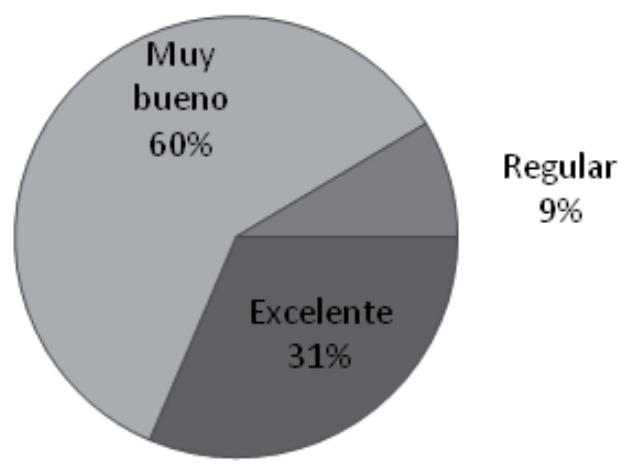

riencias concretas en investigación, así como

Como síntesis de las valoraciones obtenidas, la siguiente gráfica muestra panorámicamente el impacto alcanzado en las actividades descritas:

En resumen, este esfuerzo evaluativo debe ser considerado por la REDIECH como un insumo para la toma de decisiones en posteriores actividades. Implica considerar la crítica con ánimo constructivo y plantearse mejorar el proceso de formación que ofrece la Red a través del Foro de Investigación Educativa. La fortaleza principal es la buena acogida que tuvo la primera jornada y las expectativas que se abrieron para las posteriores.

inquietudes respecto a la importancia de la teoría y precisiones sobre los paradigmas y estrategias metodológicas en investigación educativa.

Lo que más se valora de esta actividad es que "la organización en estos grupos de trabajo permitió compartir las impresiones con otros compañeros del Foro". También se señala la dificultad de trabajar una temática tan abstracta en este formato participativo. Además las condiciones del espacio in-

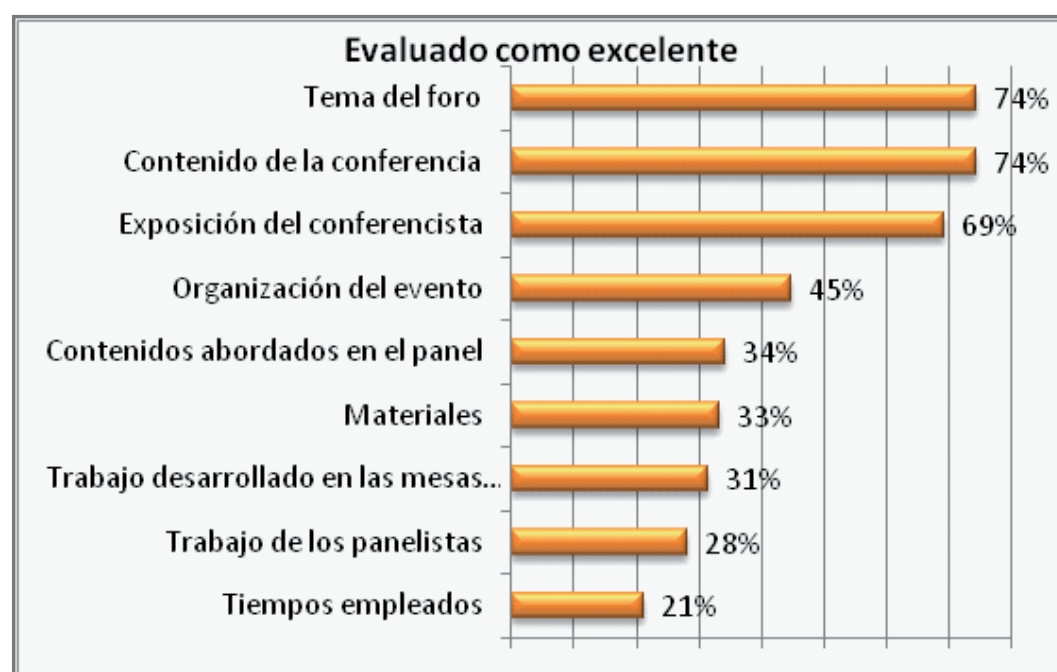

$0 \% 10 \% 20 \% 30 \% 40 \% 50 \% 60 \% 70 \% 80 \%$ terfirieron los trabajos, el ruido, sobre todo, limitó la concentración.

Respecto de las opiniones vertidas en el cuestionario sobre esta actividad, el 31\% de los asistentes opinó que la actividad fue excelente, un $60 \%$ la consideró muy buena y para el 9\% fue regular. La siguiente gráfica lo expresa con

\section{Bibliografía}

Gobantes Ollero, José María (2001). Evaluación para la mejora de la calidad educativa: planteamiento y estudio de la metodología de un caso. Revista Mexicana de Investigación Educativa, Vol. 19, No. 1, p.p. 83-104. Consultado el 01 de septiembre de 2010 en: http://revistas.um.es/rie/issue/view/8061/showToc claridad. 